\title{
Trajectory Tracking Control of Underactuated Autonomous Underwater Vehicle in the Presence of Ocean Currents
}

\author{
Lukun Wang*, Chunpeng Tian, and Xiaodong Yang \\ Department of Information Engineering, Shandong University of Science and Technology, Taian, 271019, China
}

\begin{abstract}
This paper explores trajectory tracking control for underactuated Autonomous Underwater Vehicles (AUV). The controller of dynamics based on backstepping technology is proposed. Firstly, the dynamics and kinematic model of AUV are designed. Secondly, the cascade method is applied to decompose the tracking error system into position tracking error subsystem and heading angle tracking error subsystem. Finally, backstepping technology is used to design the controller. Two virtual feedback variables are constructed, an AUV trajectory tracking controller is designed, and then the stability of the system is proven by the Lyapunov method. The results demonstrate that the proposed control schemes can make tracking errors converge uniformly.
\end{abstract}

Keywords: underactuated underwater autonomous vehicle; cascaded theory; trajectory tracking control; backstepping; Lyapunov

(Submitted on December 12, 2018; Revised on January 14, 2019; Accepted on February 16, 2019)

(C) 2019 Totem Publisher, Inc. All rights reserved.

\section{Introduction}

In recent years, the Autonomous Underwater Vehicle (AUV) as a typical nonlinear system has been widely concerned. AUV is widely applied to oil exploration, deep sea survey, ocean search, etc. [1-4]. Compared with full-actuated AUV, the underactuated AUV is subject to the restriction of nonholonomic constraints [5], and the actual power input of underactuated AUV is less than the number of freedom degrees. The trajectory planning and trajectory control of AUV has become a research hotspot. In recent years, many research works have proposed AUV trajectory control. Wang et al. [6] proposed an incremental controller to realize the tracking control of AUV. Miao et al. [7] adopted the adaptive neural network to create the trajectory tracking control law, which was founded on the dynamic surface control theory. The RBF network was used to track the trajectory of AUV [8]. Subudhi et al. [9] introduced a feedback controller to operate AUV desired tracking. The tracking controller based on the backstepping method was presented [10], which was established on the theory of virtual vehicles. In the case of influence of ocean currents, Caharija et al. [11] designed the adaptive feedback controller to follow the AUV trajectory. Do et al. [12] used the S-F coordinate system to build the error equation of path tracking. The tracking parameters were applied as the control variables, and the corresponding tracking controller was presented. Borhaug et al. [13] studied the AUV line tracking problem in three-dimensional space.

In the practical applications of underactuated AUV, ignoring the interference of ocean currents often leads to great deviations. Therefore, this paper studies the trajectory tracking control of underactuated AUV under the influence of weak currents based on the cascade system theory. The dynamic and kinematic model of AUV under the impact of weak current are set up. Based on the cascade system theory, the error of tracking system is split into subsystems: position tracking and heading angle tracking subsystems. We design a backstepping dynamics controller.

\section{Problem Description}

Taking into account the fact that AUV is a typical nonlinear dynamic system, it is convenient to describe its motion characteristics by establishing the inertial coordinate $E-\xi \eta \zeta$ and body-fixed coordinate $B-x y z$, as shown in Figure 1 .

\footnotetext{
* Corresponding author.

E-mail address: wanglukun@gmail.com
} 


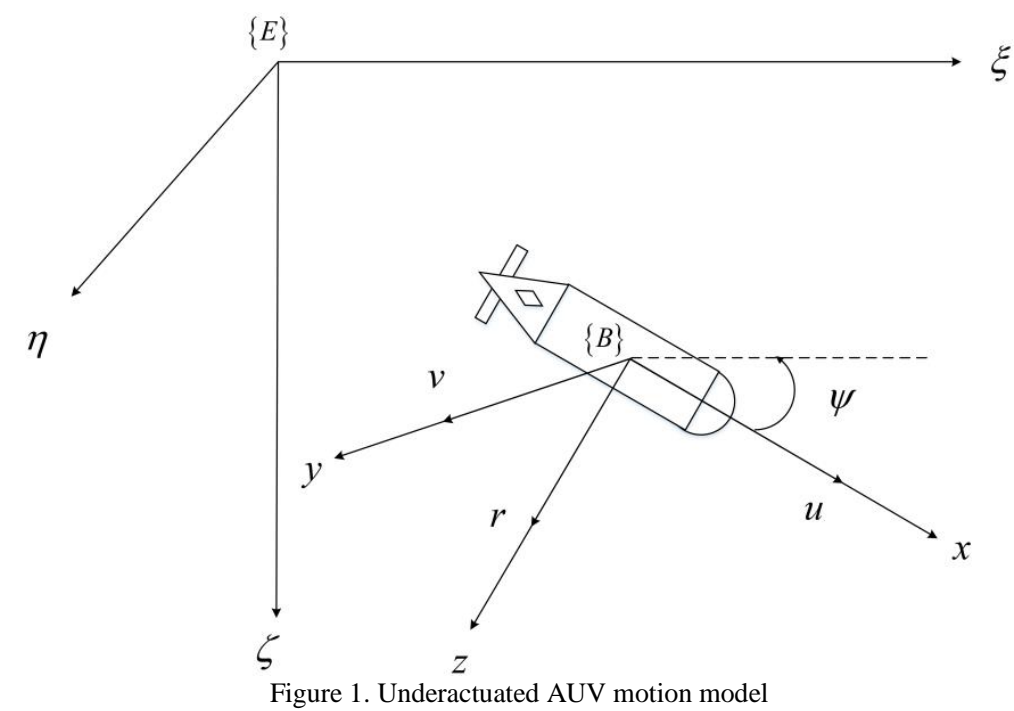

We assume the underactuated AUV moves on the horizontal. Neglecting the roll motion and the coupling reaction between the horizontal and vertical plane, the kinematic equation and dynamics equation [14] under the influence of weak current are established.

$$
\begin{gathered}
\left\{\begin{array}{l}
\dot{x}=u \cos \psi-v \sin \psi+V_{c x} \\
\dot{y}=u \sin \psi+v \cos \psi+V_{c y} \\
\dot{\psi}=r
\end{array}\right. \\
\left\{\begin{array}{l}
m_{u} \dot{u}-m_{v} v r+d_{u} u=\tau_{u} \\
m_{v} \dot{v}+m_{u} u r+d_{v} v=0 \\
m_{r} \dot{r}-m_{u v} u v+d_{r} r=\tau_{r}
\end{array}\right.
\end{gathered}
$$

Where $(x, y)$ is the coordinate in horizontal plane, $\psi$ is the heading angle, $r$ is the heading angular velocity, $u$ is the longitudinal velocity of AUV, $v$ refers to the lateral velocity of AUV with respect to the water, $\tau_{u}$ represents the longitudinal driving force provided by the rear propeller of AUV, $\tau_{r}$ means the external torque, and $V_{c x}$ and $V_{c y}$ respectively indicate the components of current velocity along the horizontal axis and longitudinal axis in the coordinate $\{B\}$. The relevant parameters in Equation (2) satisfy the following conditions.

$$
\begin{gathered}
\left\{\begin{array}{l}
m_{u}=m-X_{u} \\
m_{v}=m-Y_{\dot{v}} \\
m_{r}=I_{z}-N_{\dot{r}} \\
m_{u v}=m_{u}-m_{v}
\end{array}\right. \\
\left\{\begin{array}{l}
d_{u}=-X_{u}-X_{|u| u} \\
d_{v}=-Y_{v}-Y_{|v| v} \\
d_{r}=-N_{r}-N_{|r| r}
\end{array}\right.
\end{gathered}
$$

Where $m$ refer to mass; $I_{z}$ represents the moment of inertia; $X_{\dot{u}}, Y_{\dot{v}}$, and $N_{\dot{r}}$ refer to the hydrodynamic; and $X_{u}, Y_{v}$, $N_{r}, X_{|| \mid u}, Y_{|v| v}$, and $N_{|r| r}$ characterize the hydrodynamic damping coefficients. 
Assume that the reference trajectory satisfies

$$
\begin{gathered}
\left\{\begin{array}{l}
\dot{x}_{d}=u_{d} \cos \psi_{d}-v_{d} \sin \psi_{d} \\
\dot{y}_{d}=u_{d} \sin \psi_{d}+v_{d} \cos \psi_{d} \\
\dot{\psi}_{d}=r_{d}
\end{array}\right. \\
\left\{\begin{array}{l}
m_{u} \dot{u}_{d}-m_{v} v_{d} r_{d}+d_{u} u_{d}=\tau_{u_{d}} \\
m_{v} \dot{v}_{d}+m_{u} u_{d} r_{d}+d_{v} v_{d}=0 \\
m_{r} \dot{r}_{d}-m_{u v} u_{d} v_{d}+d_{r} r_{d}=\tau_{r_{d}}
\end{array}\right.
\end{gathered}
$$

Where $\left(x_{d}, y_{d}\right)$ is the reference coordinate of $\mathrm{AUV}, \psi_{d}$ is the reference heading angle, $r_{d}$ is the reference heading angular velocity, $u_{d}$ denotes the reference longitudinal velocity, and $v$ is the reference lateral velocity.

The tracking error of inertial coordinate $\{E\}$ can be obtained by projecting the path tracking error to the body-fixed coordinate $\{B\}$.

$$
\left[\begin{array}{l}
x_{e} \\
y_{e} \\
\psi_{e}
\end{array}\right]=\left[\begin{array}{ccc}
\cos \psi & \sin \psi & 0 \\
-\sin \psi & \cos \psi & 0 \\
0 & 0 & 1
\end{array}\right]\left[\begin{array}{c}
x-x_{d} \\
y-y_{d} \\
\psi-\psi_{d}
\end{array}\right]
$$

The velocity error can be calculated as

$$
\left\{\begin{array}{l}
u_{e}=u-u_{d} \\
v_{e}=v-v_{d} \\
r_{e}=r-r_{d}
\end{array}\right.
$$

Differentiating (7), a kinematic tracking error differential equation can be obtained as

$$
\left\{\begin{array}{l}
\dot{x}_{e}=r_{e} y_{e}+r_{d} y_{e}+u_{e}+\left(1-\cos \psi_{e}\right) u_{d}-\sin \psi_{e} v_{d}+V_{c x} \\
\dot{y}_{e}=-r_{e} x_{e}-r_{d} x_{e}+v_{e}+\sin \psi_{e} u_{d}+\left(1-\cos \psi_{e}\right) v_{d}+V_{c y} \\
\dot{\psi}=r_{e}
\end{array}\right.
$$

Substituting (2) and (6) into the differentiation of (8), we can get a dynamics tracking error differential equation.

$$
\left\{\begin{array}{l}
\dot{u}_{e}=\frac{m_{v}}{m_{u}}\left(v r-v_{d} r_{d}\right)-\frac{d_{u}}{m_{u}} u_{e}+\frac{1}{m_{u}}\left(\tau_{u}-\tau_{u_{d}}\right) \\
\dot{v}_{e}=-\frac{m_{u}}{m_{v}}\left(u r-u_{d} r_{d}\right)-\frac{d_{v}}{m_{v}} v_{e} \\
\dot{r}_{e}=\frac{m_{u}-m_{v}}{m_{r}}\left(u v-u_{d} v_{d}\right)-\frac{d_{r}}{m_{r}} r_{e}+\frac{1}{m_{r}}\left(\tau_{r}-\tau_{r_{d}}\right)
\end{array}\right.
$$

\section{Design of Trajectory Tracking Controller}

We decompose the tracking error into two subsystems by using the cascade theory. The Lyapunov function and virtual control of each subsystem are set up by using the integral backstepping method, and the underactuated AUV controller can be designed. 
Consider the cascade system:

$$
\left\{\begin{array}{l}
\dot{x}_{1}=f_{1}\left(t, x_{1}\right)+G\left(t, x_{1}, x_{2}\right) x_{2} \\
\dot{x}_{2}=f_{2}\left(t, x_{2}\right)
\end{array}\right.
$$

Where $x_{1} \in R^{n}, x_{2} \in R^{m}, f_{1}\left(t, x_{1}\right)$ is continuously differentiable, and $f_{2}\left(t, x_{2}\right)$ and $G\left(t, x_{1}, x_{2}\right)$ can satisfy the local Lipschitz condition at the points of $x_{2}$ and $\left(x_{1}, x_{2}\right)$ respectively.

Suppose the reference angular velocity $r_{d}$ of AUV is a constant. According to Equation (11), the underactuated AUV tracking error systems (9) and (10) can be decomposed into two subsystems. The position vector $\boldsymbol{x}_{1}$ and angle vector $\boldsymbol{x}_{2}$ can be defined as

$$
\boldsymbol{x}_{1}=\left[\begin{array}{llll}
x_{e} & y_{e} & u_{e} & v_{e}
\end{array}\right]^{T}, \boldsymbol{x}_{2}=\left[\begin{array}{ll}
\psi_{e} & r_{e}
\end{array}\right]^{T}
$$

The position tracking error subsystem can be defined as

$$
f_{1}\left(t, \boldsymbol{x}_{1}\right)=\left\{\begin{array}{l}
\dot{x}_{e}=r_{d} y_{e}+u_{e}+V_{c x} \\
\dot{y}_{e}=-r_{d} x_{e}+v_{e}+V_{c y} \\
\dot{u}_{e}=\frac{m_{v}}{m_{u}} v_{e} r_{d}-\frac{d_{u}}{m_{u}} u_{e}+\frac{1}{m_{u}}\left(\tau_{u}-\tau_{u_{d}}\right) \\
\dot{v}_{e}=-\frac{m_{u}}{m_{v}} u_{e} r_{d}-\frac{d_{v}}{m_{v}} v_{e}
\end{array}\right.
$$

The related item of the position subsystem is

$$
G\left(t, \boldsymbol{x}_{1}, \boldsymbol{x}_{2}\right)=\left[\begin{array}{cc}
\frac{1-\cos \psi_{e}}{\psi_{e}} u_{d}-\frac{\sin \psi_{e}}{\psi_{e}} v_{d} & y_{e} \\
\frac{\sin \psi_{e}}{\psi_{e}} u_{d}+\frac{1-\cos \psi_{e}}{\psi_{e}} v_{d} & -x_{e} \\
0 & \frac{m_{v}}{m_{u}}\left(v_{e}+v_{d}\right) \\
0 & -\frac{m_{u}}{m_{v}}\left(u_{e}+u_{d}\right)
\end{array}\right]
$$

The heading angel tracking error subsystem would be

$$
f_{2}\left(t, \boldsymbol{x}_{2}\right)=\left\{\begin{array}{l}
\dot{\psi}_{e}=r_{e} \\
\dot{r}_{e}=\frac{m_{u}-m_{v}}{m_{r}}\left(u v-u_{d} v_{d}\right)-\frac{d_{r}}{m_{r}} r_{e}+\frac{1}{m_{r}}\left(\tau_{r}-\tau_{r_{d}}\right)
\end{array}\right.
$$

According to the model of AUV, a virtual error $\bar{x}_{e}$ for subsystem (12) is defined as

$$
\bar{x}_{e}=x_{e}-k_{1} r_{d} y_{e}-\frac{V_{c y}}{r_{d}}
$$

Where $k_{1}>0$, and the Lyapunov function is defined as 


$$
V_{1}=\frac{1}{2} \bar{x}_{e}^{2}+\frac{1}{2} y_{e}^{2}+\frac{m_{v}}{2} v_{e}^{2}
$$

Differentiating (16), we obtain

$$
\begin{aligned}
\dot{V}_{1} & =\bar{x}_{e}\left(r_{d} y_{e}+u_{e}+V_{c x}+k_{1} r_{d}^{2} x_{e}-k_{1} r_{d} v_{e}-k_{1} r_{d} V_{c y}\right)+y_{e}\left(-r_{d} x_{e}+v_{e}+V_{c y}\right)+m_{v} v_{e}\left(-\frac{m_{u}}{m_{v}} u_{e} r_{d}-\frac{d_{v}}{m_{v}} v_{e}\right) \\
& =\bar{x}_{e}\left(r_{d} y_{e}+u_{e}+V_{c x}+k_{1} r_{d}^{2} \bar{x}_{e}+k_{1} r_{d}^{3} y_{e}-k_{1} r_{d} v_{e}\right)+y_{e}\left(-r_{d} \bar{x}_{e}-k_{1} r_{d}^{2} y_{e}+v_{e}\right)+m_{v} v_{e}\left(-\frac{m_{u}}{m_{v}} u_{e} r_{d}-\frac{d_{v}}{m_{v}} v_{e}\right) \\
& =-k_{1} r_{d}^{2} y_{e}^{2}-d_{v} v_{e}^{2}+k_{1} r_{d}^{2} \bar{x}_{e}^{2}+y_{e} v_{e}+\bar{x}_{e}\left(k_{1} r_{d}^{3} y_{e}+u_{e}-k_{1} r_{d} v_{e}+V_{c x}\right)-m_{u} r_{d} v_{e} u_{e}
\end{aligned}
$$

A virtual error $\bar{u}_{e}$ is defined as

$$
\bar{u}_{e}=u_{e}+k_{2} \bar{x}_{e}
$$

Where $k_{2}>0$. Substituting (18) into (17), we obtain

$$
\begin{aligned}
& \dot{V}_{1}=-k_{1} r_{d}^{2} y_{e}^{2}-d_{v} v_{e}^{2}+k_{1} r_{d}^{2} \bar{x}_{e}^{2}+y_{e} v_{e}+\bar{x}_{e}\left(k_{1} r_{d}^{3} y_{e}+u_{e}-k_{1} r_{d} v_{e}+V_{c x}\right)-m_{u} r_{d} v_{e} u_{e} \\
& =-k_{1} r_{d}^{2} y_{e}^{2}-d_{v} v_{e}^{2}-\left(k_{2}-k_{1} r_{d}^{2}\right) \bar{x}_{e}^{2}+\bar{x}_{e}\left(k_{1} r_{d}^{3} y_{e}+\left(k_{2} m_{u} r_{d}-k_{1} r_{d}\right) v_{e}+V_{c x}\right)+\bar{u}_{e}\left(\bar{x}_{e}-m_{u} r_{d} v_{e}\right)
\end{aligned}
$$

Let the Lyapunov function be defined as

Differentiating (20), we obtain

$$
V_{2}=V_{1}+\frac{m_{u}}{2} \bar{u}_{e}^{2}
$$

$$
\begin{aligned}
\dot{V}_{2}= & -k_{1} r_{d}^{2} y_{e}^{2}-d_{v} v_{e}^{2}-\left(k_{2}-k_{1} r_{d}^{2}\right) \bar{x}_{e}^{2}+\bar{x}_{e}\left[k_{1} r_{d}^{3} y_{e}+\left(k_{2} m_{u} r_{d}-k_{1} r_{d}\right) v_{e}+V_{c x}\right]+\bar{u}_{e}\left(\bar{x}_{e}-m_{u} r_{d} v_{e}\right) \\
& +\bar{u}_{e}\left[m_{v} v_{e} r_{d}-d_{u} u_{e}+\left(\tau_{u}-\tau_{u_{d}}\right)+k_{2}\left(r_{d} y_{e}+u_{e}+V_{c x}+k_{1} r_{d}^{2} \bar{x}_{e}+k_{1}^{2} r_{d}^{3} y_{e}-k_{1} r_{d} v_{e}\right)\right] \\
= & -k_{1} r_{d}^{2} y_{e}^{2}-d_{v} v_{e}^{2}-\left(k_{2}-k_{1} r_{d}^{2}\right) \bar{x}_{e}^{2}-\left(d_{u}-k_{2}\right) \bar{u}_{e}^{2}+\bar{x}_{e}\left[k_{1} r_{d}^{3} y_{e}+\left(k_{2} m_{u} r_{d}-k_{1} r_{d}\right) v_{e}+V_{c x}\right] \\
& +\bar{u}_{e}\left[\bar{x}_{e}\left(1+k_{2} d_{u}-k_{2}^{2}+k_{1}^{2} k_{2} r_{d}^{2}\right)+k_{2} V_{c x}+y_{e}\left(k_{2} r_{d}+k_{1}^{2} k_{2} r_{d}^{3}\right)-k_{1} k_{2} r_{d} v_{e}+\left(\tau_{u}-\tau_{u_{d}}\right)\right]
\end{aligned}
$$

The control input $\tau_{u}$ is selected as

$$
\tau_{u}=\tau_{u_{d}}-\bar{x}_{e}\left(1+k_{2} d_{u}-k_{2}^{2}+k_{1}^{2} k_{2} r_{d}^{2}\right)-y_{e}\left(k_{2} r_{d}+k_{1}^{2} k_{2} r_{d}^{3}\right)+k_{1} k_{2} r_{d} v_{e}-k_{2} V_{c x}
$$

Substituting (21) into (22), we obtain

$$
\dot{V}_{2}=-k_{1} r_{d}^{2} y_{e}^{2}-d_{v} v_{e}^{2}-\left(k_{2}-k_{1} r_{d}^{2}\right) \bar{x}_{e}^{2}-\left(d_{u}-k_{2}\right) \bar{u}_{e}^{2}+\bar{x}_{e}\left[k_{1} r_{d}^{3} y_{e}+\left(k_{2} m_{u} r_{d}-k_{1} r_{d}\right) v_{e}+V_{c x}\right]
$$

In order to facilitate the derivation, the parameters need to satisfy

$$
k_{2} m_{u} r_{d}-k_{1} r_{d}=0
$$

By (23), we get 


$$
\begin{aligned}
\dot{V}_{2}= & -k_{1} r_{d}^{2} y_{e}^{2}-d_{v} v_{e}^{2}-\left(k_{2}-k_{1} r_{d}^{2}\right) \bar{x}_{e}^{2}-\left(d_{u}-k_{2}\right) \bar{u}_{e}^{2}+k_{1} r_{d}^{3} y_{e} \bar{x}_{e}+\bar{x}_{e} V_{c x} \\
= & -\left(\sqrt{\frac{k_{2}-k_{1} r_{d}^{2}}{2}} \bar{x}_{e}-\frac{k_{1} r_{d}^{3}}{\sqrt{2\left(k_{2}-k_{1} r_{d}^{2}\right)}} y_{e}\right)^{2}-\frac{\left(k_{2}-k_{1} r_{d}^{2}\right)}{2} \bar{x}_{e}^{2} \\
& -\left(k_{1} r_{d}^{2}-\frac{k_{1}^{2} r_{d}^{6}}{2\left(k_{2}-k_{1} r_{d}^{2}\right)}\right) y_{e}^{2}-d_{v} v_{e}^{2}-\left(d_{u}-k_{2}\right) \bar{u}_{e}^{2}+\bar{x}_{e} V_{c x}
\end{aligned}
$$

If $\left\|\bar{x}_{e}\right\|<2 V_{c x} /\left(k_{2}-k_{1} r_{d}^{2}\right)$, one gets

$$
-\frac{\left(k_{2}-k_{1} r_{d}^{2}\right)}{2} \bar{x}_{e}^{2}+\bar{x}_{e} V_{c x}<0
$$

In order to ensure $\dot{V}_{2}<0$, the parameters need to satisfy

$$
k_{1} r_{d}^{2}-\frac{k_{1}^{2} r_{d}^{6}}{2\left(k_{2}-k_{1} r_{d}^{2}\right)}>0, k_{2}<d_{u}
$$

The stability theorem of the position tracking error subsystem (12) is thus given.

Theorem 1 Tracking error subsystem (12) and related term (13) will asymptotically converge if controller (22) is applied, and relevant parameters can satisfy the requirements of (24), (26), and (27).

For subsystem (14), the Lyapunov function can be defined.

$$
V_{3}=\frac{1}{2} \psi_{e}^{2}+\frac{1}{2} r_{e}^{2}
$$

Differentiating (28), we obtain

$$
\begin{aligned}
\dot{V}_{3} & =\dot{\psi}_{e} \psi_{e}+\dot{r}_{e} r_{e} \\
& =r_{e} \psi_{e}+r_{e}\left[\frac{m_{u}-m_{v}}{m_{r}}\left(u v-u_{d} v_{d}\right)-\frac{d_{r}}{m_{r}} r_{e}+\frac{1}{m_{r}}\left(\tau_{r}-\tau_{r_{d}}\right)\right] \\
& =r_{e}\left[\frac{m_{u}-m_{v}}{m_{r}}\left(u v-u_{d} v_{d}\right)-\frac{d_{r}}{m_{r}} r_{e}+\frac{1}{m_{r}}\left(\tau_{r}-\tau_{r_{d}}\right)+\psi_{e}\right]
\end{aligned}
$$

The control input $\tau_{u}$ is selected as

$$
\tau_{r}=\tau_{r_{d}}-\left(m_{u}-m_{v}\right)\left(u v-u_{d} v_{d}\right)+d_{r} r_{e}-m_{r} \psi_{e}-k_{3} r_{e}
$$

Where $k_{3}>0$. Substituting (30) into (29), we obtain

$$
\dot{V}_{3}=-k_{3} r_{e}^{2}
$$

Because $r_{e}^{2} \leq 0, \dot{V}_{3} \leq 0 . \dot{V}_{3}$ is semi-negative definite. On the basis of the Baralat lemma [15], it can be determined that $\dot{V}_{3} \rightarrow 0$ when $t \rightarrow \infty$ such that $\lim _{t \rightarrow \infty} r_{e}=0$. The stability theorem of heading angle tracking error subsystem (14) is thus given.

Theorem 2 Tracking error subsystem (14) will be asymptotically stable if tracking controller (30) is applied. 


\section{Simulation Results}

For the purpose of verifying the effectiveness of controllers (22) and (30), a simulation experiment is designed. Assume that the physical parameters of underactuated AUV are as follows: $m_{u}=63.25 \mathrm{~kg}, m_{v}=31.43 \mathrm{~kg}, m_{r}=30 \mathrm{~kg}, d_{u}=63 \mathrm{~kg} / \mathrm{s}$, $d_{v}=262 \mathrm{~kg} / \mathrm{s}$, and $d_{r}=70 \mathrm{~kg} / \mathrm{s}$. The control parameters are as follows: $k_{1}=2, k_{2}=2$, and $k_{3}=1$. The motion trajectory is set to be a circle, and we set $x_{d}(0)=3 \mathrm{~m}, y_{d}(0)=5 \mathrm{~m}, \psi_{d}(0)=\pi / 6 \mathrm{rad}, u_{d}(0)=5 \mathrm{~m} / \mathrm{s}, v_{d}(0)=0 \mathrm{~m} / \mathrm{s}$, and $r_{d}(0)=0.4 \mathrm{rad} / \mathrm{s}$. We set the initial poses $x(0)=1 \mathrm{~m}, y(0)=2 \mathrm{~m}, \psi(0)=0 \mathrm{rad}, u_{r}(0)=1 \mathrm{~m} / \mathrm{s}, v_{r}(0)=0 \mathrm{~m} / \mathrm{s}$, and $r(0)=1 \mathrm{rad} / \mathrm{s}$. The related parameters of the current are set as: $v_{c}=0.1 \mathrm{~m} / \mathrm{s}, \psi_{c}=\pi / 6 \mathrm{rad}, r_{d}=0.6 \mathrm{rad} / \mathrm{s}$, and $u_{d}=5 \mathrm{~m} / \mathrm{s}$.

Figure 2(a) is the motion trajectory curve. The actual motion and the reference trajectory are almost coincident by means of controllers (22) and (30). Figure 2(b) is the position tracking error curve. According to the results, it can be seen that the position tracking error subsystem (12) tends to be stable at 5s. Figure 2(c) is the heading angle error curve, and the error subsystem (14) is asymptotically stable at 10s. Figure 2(d) is the longitudinal driving force curve, and Figure 2(e) is the external torque curve. We can see that these two control input curves converge to a stable range. According to these results, the effectiveness can be confirmed.
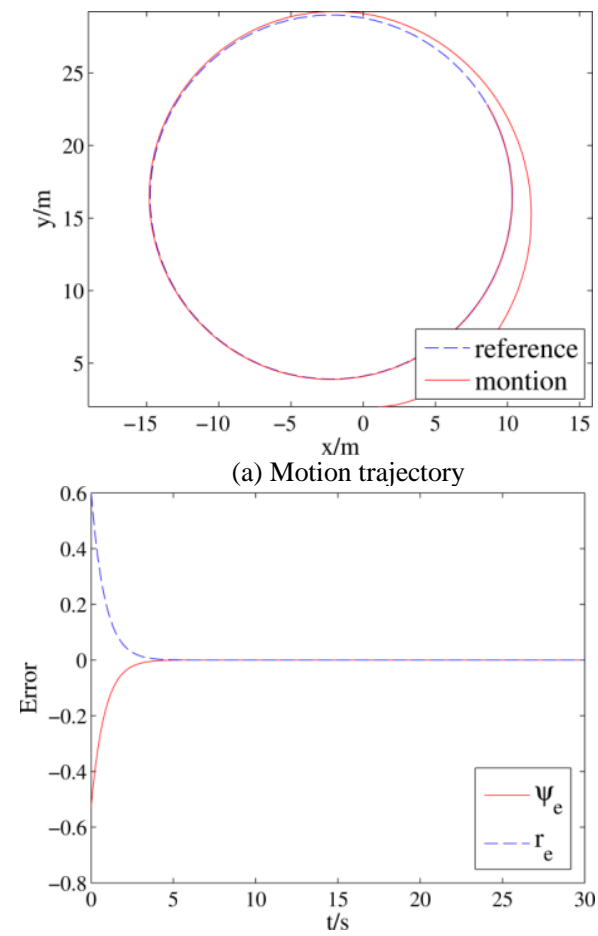

(c) Heading angle error
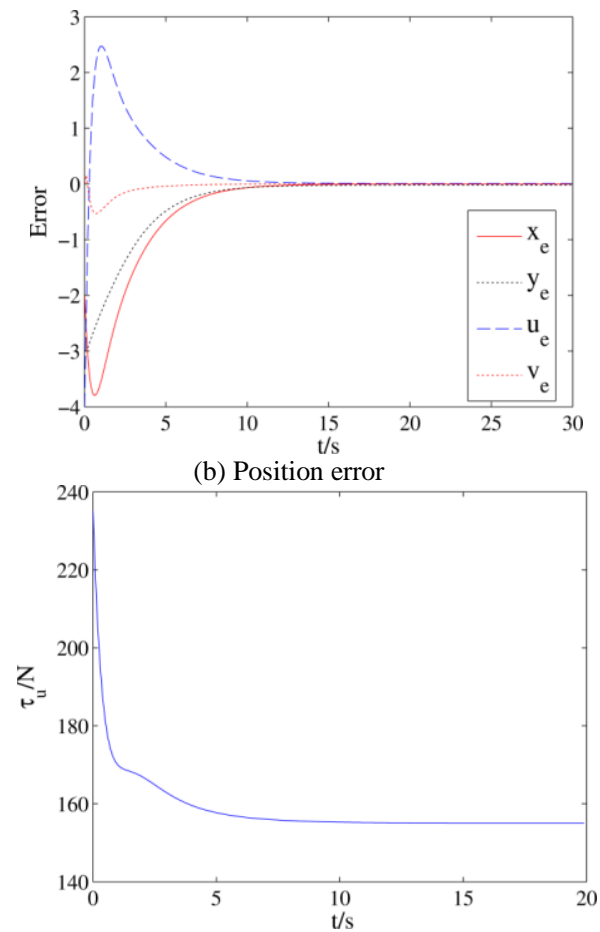

(d) Longitudinal driving force

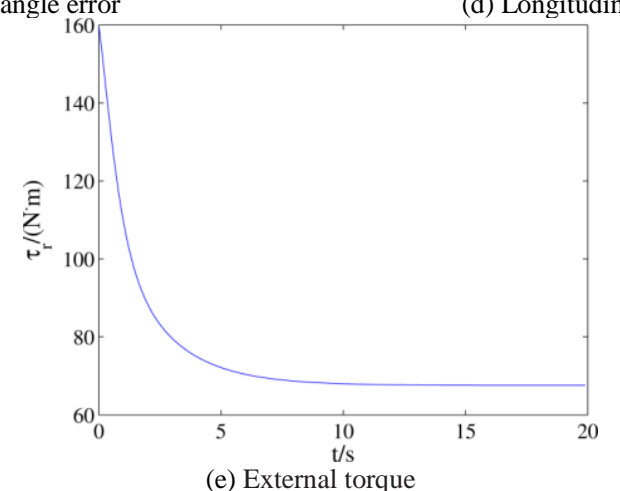

Figure 2. The curve of AUV

\section{Conclusions}

This paper has studied the trajectory tracking of underactuated AUV. The dynamics and kinematic model of underactuated 
AUV under the influence of weak current have been established. The tracking error has been decomposed into position tracking error and heading angel tracking error using the cascade method. Two virtual feedback variables have respectively been designed, combined with backstepping to propose the control law of position tracking and then the control law of heading angle tracking. Finally, experiments have been carried out for simulation. The results have demonstrated the availability of the controller designed in our paper.

\section{Acknowledgments}

This research was supported by the National Natural Science Foundation of Shandong Province (No. ZR2018BF005), the Scientific Research Foundation of Shandong University of Science and Technology for Recruited Talents (No. 2017RCJJ077), the Shandong Province Higher Educational Science and Technology Program (No. J17KB167), and the Science and Technology Program of Taian (No. 2017GX0014).

\section{References}

1. F. Repoulias and E. Papadopoulos, "Planar Trajectory Planning and Tracking Control Design for Underactuated AUVs," Ocean Engineering, Vol. 34, No. 11-12, pp. 1650-1667, 2007

2. L. Lapierre and D. Soetanto, "Nonlinear Path-Following Control of an AUV," Ocean Engineering, Vol. 34, No. 11-12, pp. 1734-1744, 2007

3. M. Pebody, “Autonomous Underwater Vehicle Collision Avoidance for under-Ice Exploration," Proceedings of the Institution of Mechanical Engineers Part M: Journal of Engineering for the Maritime Environment, Vol. 222, No. 2, pp. 53-66, 2008

4. O. Calvo, A. Rozenfeld, A. Souza, F. Valenciaga, P. F. Puleston, and G. Acosta, "Experimental Results on Smooth Path Tracking with Application to Pipe Surveying on Inexpensive AUV," in Proceedings of IEEE/RSJ International Conference on Intelligent Robots and Systems, pp. 3647-3653, Nice, France, 2008

5. A. P. Aguiar, J. P. Hespanha, and A. M. Pascoal, "Switched Seesaw Control for the Stabilization of Underactuated Vehicles," Automatica, Vol. 43, No. 12, pp. 1997-2008, 2007

6. L. Wang, L. J. Zhang, H. M. Jia, and H. B. Wang, "Horizontal Tracking Control for AUV based on Nonlinear Sliding Mode," in Proceedings of IEEE International Conference on Information and Automation, pp. 460-463, Shenyang, China, 2012

7. B. B. Miao, T. S. Li, and W. L. Luo, "A DSC and MLP based Robust Adaptive NN Tracking Control for Underwater Vehicle," Neurocomputing, Vol. 111, pp. 184-189, 2013

8. X. Q. Bian, J. J. Zhou, Z. P. Yan, and H. M. Jia, “Adaptive Neural Network Control System of Path Following for AUVs," in Proceedings of IEEE SoutheastCon, IEEE Region-3, Orlando, United States, 2012

9. B. Subudhi, K. Mukherjee, and S. Ghosh, "A Static Output Feedback Control Design for Path Following of Autonomous Underwater Vehicle in Vertical Plane," Ocean Engineering, Vol. 63, pp. 72-76, 2013

10. W. Zhang, D. Xu, M. L. Tan, C. L. Wang, and Z. P. Yan, "Trajectory Tracking Control of Underactuated UUV for Underwater Recovery," in Proceedings of the 2nd International Conference on Instrumentation, Measurement, Computer, Communication and Control, pp. 386-391, Harbin, China, 2012

11. W. Caharija, K. Y. Pettersen, J. T. Gravdahl, and E. Borhaug, "Path Following of Underactuated Autonomous Underwater Vehicles in the Presence of Ocean Currents," in Proceedings of the IEEE Conference on Decision and Control, pp. 528-535, Maui, Hawaii, USA, 2012

12. K. D. Do, J. Pan, and Z. P. Jiang, "Robust and Adaptive Path Following for Underactuated Autonomous Vehicles," Ocean Engineering, Vol. 31, No. 16, pp. 1967-1977, 2004

13. E. Borhaug and K. Y. Pettersen, "Cross-Track Control for Underactuated Autonomous Vehicles," in Proceedings of the IEEE Conference on Decision and Control, and the European Control Conference, pp. 12-15, Seville, Spain, 2005

14. A. P. Aguiar and A. M. Pascoal, "Dynamic Positioning and Way-Point Tracking of Underactuated AUVs in the Presence of Ocean Currents," International Journal of Control, Vol. 80, No. 7, pp. 1092-1108, 2007

15. R. W. Brockett, "Asymptotic Stability and Feedback Stabilization," Differential Geometric Control Theory, Vol. 27, pp. 181191, 1983

Lukun Wang received his B.S. degree in computer science from Shandong University of Science and Technology in 2006, his M.S. degree in software engineering from Dalian University of Technology in 2006, and his Ph.D. in computer science from Ocean University of China. Currently, he is a lecturer at Shandong University of Science and Technology. His current research interests include artificial intelligence, image processing and pattern recognition, machine learning, control theory and applications, and sensor networks.

Chunpeng Tian received his B.S. degree in computer science from University of Science \& Technology Beijing in 2009, his M.S. degree in computer science from Qingdao University of Science and Technology in 2012, and his Ph.D. in computer science from Ocean University of China. Currently, he is a lecturer at Shandong University of Science and Technology.

Xiaodong Yang received his B.S. degree in computer science from Fuzhou University in 1991. Currently, he is an associate professor at Shandong University of Science and Technology. 